\title{
A Degree-Based Heuristic for Strongly Connected Dominating-Absorbent Sets in Wireless Ad-Hoc Networks
}

\author{
Christine Markarian \\ Department of Computer Science and Mathematics \\ Lebanese American University \\ Beirut, Lebanon \\ Email: christine.markarian@lau.edu.lb
}

\author{
Faisal N. Abu-Khzam \\ Department of Computer Science and Mathematics \\ Lebanese American University \\ Beirut, Lebanon \\ Email: faisal.abukhzam@lau.edu.lb
}

\begin{abstract}
Virtual backbones of asymmetric wireless networks are special sub-nets through which routing can be performed. Such backbone must be as small as possible, and must be able to receive and transmit messages from/to each and every node in the network. The corresponding graph theoretic problem takes a directed graph as input and seeks a strongly connected dominating-absorbent set of smallest possible cardinality. We introduce a hybrid heuristic for this problem, in which we combine low-degree vertex elimination and high-degree vertex selection. This simple and efficient method yields very promising experimental results, outperforming known heuristic algorithms.
\end{abstract}

\section{INTRODUCTION}

Due to lack of a fixed infrastructure, communication in wireless ad-hoc networks relies on a shared medium through either single hops or multihops, often resulting in inevitable redundancy and collision. The use of virtual backbones is often proposed as a potential solution. The nodes of a said backbone become responsible for message-routing, potentially reducing the routing overhead. Such nodes must induce a connected subgraph that is accessible by every node of the network, which requires them to form a connected dominating set.

Obviously, a small (or smallest-possible) connected dominating set is preferred. The problem of finding a minimum connected dominating set has been extensively studied in unit disc graphs (UDG), which model networks with uniform transmission range. In practice, however, the transmission ranges of nodes in the network need not be the same, due to differences in power and functionality. Therefore, a directed graph would best model such a network. Consequently, a reliable virtual backbone consists of a strongly connected dominating set such that every node can transmit and receive messages through the backbone. Such sets are called dominating and absorbing, or absorbent, sets.

Motivated by the above, we consider the Strongly Connected Dominating-Absorbent Set problem, hence- forth SCDAS, in general directed graphs. SCDAS is formally defined as follows:

Given: A digraph $G=(V, E)$

Question: Find $D \subset V$ of minimum cardinality such that $D$ is dominating and absorbent and the subgraph induced by $D$ is strongly connected.

SCDAS is NP-Complete, by a simple reduction from Connected Dominating Set. So far, the only exact algorithm is the trivial brute-force enumeration. The only work on SCDAS has thus far focused on heuristic approaches that target time-efficiency, knowing that constant-factor approximations are hard to obtain [1], [2], [3], [4], [5], [6]. We present a new heuristic algorith$\mathrm{m}$ that favors high-degree vertices when selecting members of the target dominating set. Despite its simplicity, our algorithm delivers solutions that are much closer to optimum solutions than previously published algorithms.

The rest of this paper consists of some preliminaries and literature review, followed by a description of our algorithm (section 3). In section 4 we present experimental results that show the surprising effect of our simple solution. We conclude with some future research directions.

\section{PREliminaries}

We adopt common graph theoretic terminology and notation, such as the notions of degree, neighborhood, adjacency and incidence. For a pair $\{u, v\}$ of vertices of a digraph $G$, we say $v$ is an out-neighbor of $u$, and $u$ is an in-neighbor of $v$, whenever $u v$ is a $\operatorname{arc}$ of $G$.

The out-neighborhood of a vertex $u$, denoted by $N^{+}(u)$ is the set of out-neighbors of $u$. The inneighborhood, $N^{-}(u)$ is defined analogously. The outneighbors (respectively in-neighbors) of a set $S$ of vertices is $\left(\cup_{v \in S} N^{+}(v)\right) \backslash S$ (respectively $\left(\cup_{v \in S} N^{-}(v)\right) \backslash$ $S$ ). A set $D$ is a dominating (resp. absorbent) set if its 
complement coincides with its set of out-neighbors (resp. in-neighbors).

A directed graph is said to be strongly connected if, for any pair $\{u, v\}$ of its vertices, there is a path from $u$ to $v$ and a path from $v$ to $u$. A set of vertices is strongly connected if it induces a strongly connected subgraph. We denote by $G[D]$ the subgraph induced by a set $D$ of vertices.

A graph $G=(V, E)$ is a disk graph if its vertices are in one-to-one correspondence with disks in a plane such that an edge, or arc, from a vertex $u$ to vertex $v$ exists whenever $v$ lies in the interior of $u$. Such graphs model asymmetric wireless network since the radius of a disk corresponds to its transmission range: node $i$ can send messages to node $j$ if the center of disk $j$ is in the interior of disk $i$. Note that message sending is not symmetric since the center of $i$ need not be contained in the interior of $j$. Therefore, the corresponding graph is directed.

Previous work on virtual backbones of wireless networks concentrated more on symmetric networks [8], [9], [10], [11], [12]. The corresponding graphs are undirected unit-disk graphs, which enjoy special structures that lead to effective approximation algorithms. As pointed out in the previous section, we focus mainly on asymmetric networks, in which we shall seek minimal strongly connected dominating-absorbent sets. Again, the corresponding optimization problem is SCDAS.

There are a number of known algorithms for SCDAS, of which we mention some notable ones. In [1], Wu et al. presented an algorithm that is based on the following idea:

Iteratively consider nodes of the graph. If a node has two neighbors that are not joined by an arc, it becomes part of the solution, i.e. in the strongly connected dominating-absorbent set. This same approach was later improved by addition of more reduction rules to decrease the number of nodes in the constructed set [2]. No approximation ratio has been given, however. In [7], $\mathrm{Li}$ et al introduced an approximation algorithm, with a ratio bound of $3 \log n$.

More recently, Thai et al. gave two heuristics (in [4]):

1) Dominating Absorbent Spanning Trees (DAST), and

2) Greedy Strongly Connected Component Merging algorithm (G-CMA).

DAST constructs two spanning trees and outputs the union of the two trees as a strongly connected dominating-absorbent set. G-CMA finds a dominating absorbent set then uses additional nodes to make the set strongly connected via shortest paths between strongly connected components.

To the best of our knowledge, DAST and G-CMA are the most recent approaches, and they seem to have the best (published) performance. We shall show that a simple approach can successfully produce smaller solutions in practice. Our approach is a hybrid heuristic combining low-degree vertex elimination and high-degree vertex selection and we shall refer to it as LDHD in the sequel.

\section{A Vertex-Degree Based Heuristic}

A high-degree vertex is more likely to dominate and absorb other vertices than vertices of lower degree. Thus, a "good" solution will most likely contain many highdegree vertices. However, since the solution set must not only dominate and absorb all vertices in the graph, but also be strongly connected, the above statement would not be enough. Despite their disability to absorb and dominate relatively many vertices, low-degree vertices might be used as intermediary vertices to strongly connect the vertices in an optimal solution set.

Rather than discovering the low-degree vertices that contribute in the strong connectivity property, our algorithm starts by deleting vertices that do not contribute to the strong connectivity property. This can easily be done by removing a low-degree vertex from the graph and checking if the graph remains strongly connected. If so, the just removed vertex can be deleted (i.e., not counted in the solution). Moreover, when such a vertex is deleted, it has to be dominated and absorbed by a subset of the remaining vertices. Therefore, high-degree in- and out-neighbors are selected (i.e., added to the solution) to dominate and absorb the deleted vertex. Once a vertex is selected, it can never be deleted.

In the following formal description of LDHD, the vertices are colored green, red or white. A green vertex is one that is decided to be in the solution, while a red one is a deleted vertex (decided not to be in). A white vertex is not yet decided. Initially, all vertices are white and, at any stage the white and green vertices induce a strongly connected dominating-absorbent set (initially, all vertices must form a strongly-connected dominating-absorbent set). The algorithm ends when no white vertices remain.

In addition to the above procedure, when the number of non-red in-neighbors (or out-neighbors) of a white vertex $v$ drops to one, the only one in-neighbor (or outneighbor) of $v$ is automatically placed in the solution (if not already green), being the only vertex that can absorb (or, respectively, dominate) $v$. 


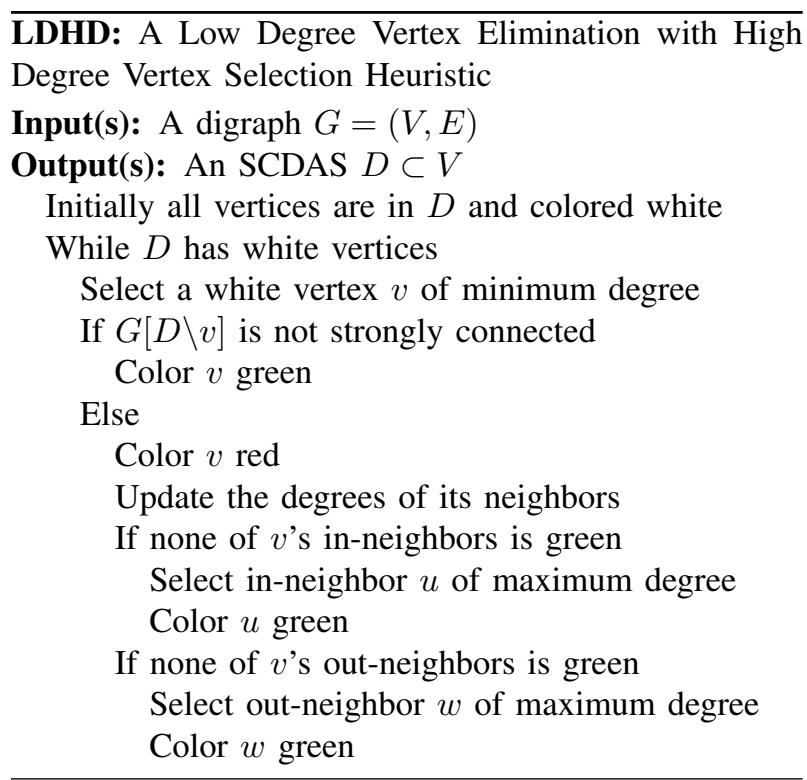

The while loop in the algorithm is executed at most $n-1$ times because at each step we are either eliminating or keeping at least one node. To check if removing a vertex disconnects the graph requires running either Depth First Search or Breadth First Search twice. This takes $O(m+n)$ time, where $m$ is the number of edges. Thus, LDHD has an overall time complexity of $O(m n)$.

\section{EXPERIMENTAL RESULTS}

We compare the performance of the following four approaches for SCDAS. The size of a produced solution is used as a quality measure.

1) Dominating Absorbent Spanning Trees: DAST

2) Greedy Strongly Connected Component Merging algorithm: G-CMA

3) A brute force optimization algorithm : EXACT

4) Our proposed heuristic: LDHD

To generate a random directed graph, we follow the same approach adopted in previously published work (see, for example, [4]). $N$ nodes with distinct identity numbers between 1 and $N$ are located (randomly) in a limited square area in the Euclidean plane. Each node chooses a random transmission range that is bounded by some specified minimum and maximum values. A directed edge is added from a node $u$ to node $v$ if the Euclidean distance between $u$ and $v$ is less than the transmission range of $u$. If the generated graph is strongly connected, we use it as one instance, otherwise we discard it.

We measure the performance of each approach under the effect of two network parameters:
1) Network Density

We vary network density in two ways:

a) Different numbers of nodes in a fixed area

b) Different area sizes for a fixed number of nodes

2) Transmission ratio: $k=T_{r \max } / T_{r \min }$, where $T_{r \max }$ and $T_{r \min }$ are the maximum and minimum transmission ranges respectively.

Simulation for each performance measure is repeated 100 times for each instance and the average result is taken.

\section{A. Network Density: Different Number of Nodes}

To compare the size of the strongly connected dominating-absorbent sets constructed by each of the four approaches, we deploy $N$ nodes in a $1000 \mathrm{~m} \mathrm{x}$ $1000 \mathrm{~m}$ area. $N$ changes between 10 and 130 with an increment of 10. The nodes select their transmission ranges from the interval $\left[T_{r \min }, T_{r \max }\right]$.

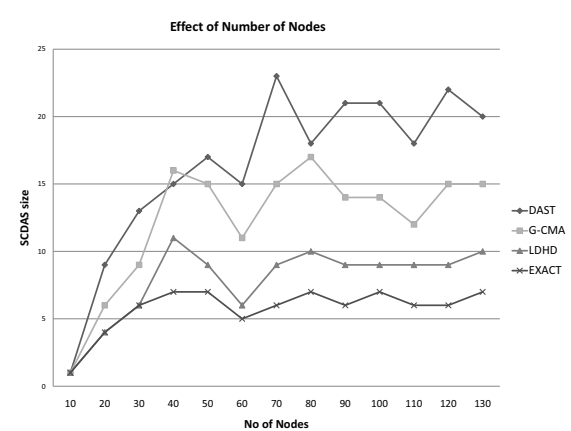

Fig. 1. Network Density: Different number of nodes

\section{B. Network Density: Different area size}

To study the effect of varying the area on the performance of each approach, we deploy a fixed number of nodes, $N=50$. The nodes select their transmission ranges from the interval $\left[T_{r \min }, T_{r \max }\right]$. The area varies from $600 \mathrm{~m} \times 600 \mathrm{~m}$ to $1400 \mathrm{~m} \times 1400 \mathrm{~m}$.

\section{Transmission ratio}

We also study the effect of varying the transmission ratio, $k=T_{r \max } / T_{r \min }$ on the size of the strongly connected dominating-absorbent sets constructed by each of the four approaches.

We conducted two experiments. In the first, we randomly locate 50 nodes in a fixed $1000 \mathrm{~m} \times 1000 \mathrm{~m}$ area and vary $k$ varies as follows: We fix $T_{r \max }=1000 \mathrm{~m}$ and vary $T_{r \min }$ between $200 \mathrm{~m}$ and $1000 \mathrm{~m}$ with an increment of 200 for $k=1$ to 5 . 


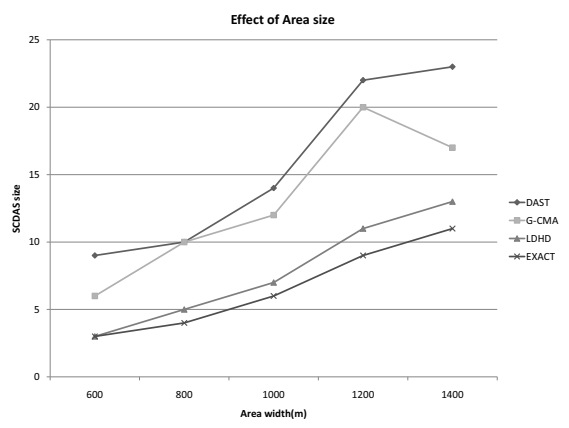

Fig. 2. Network Density: Different area size

In the second experiment, we measure the performances on a larger network and randomly locate 100 nodes in a fixed $1200 \mathrm{~m} \times 1200 \mathrm{~m}$ area and vary $k$ as follows: we fix $T_{r \max }=1200 \mathrm{~m}$ and vary $T_{r \min }$ between $200 \mathrm{~m}$ and $1200 \mathrm{~m}$ with an increment of 200 for $k=1$ to 6 .

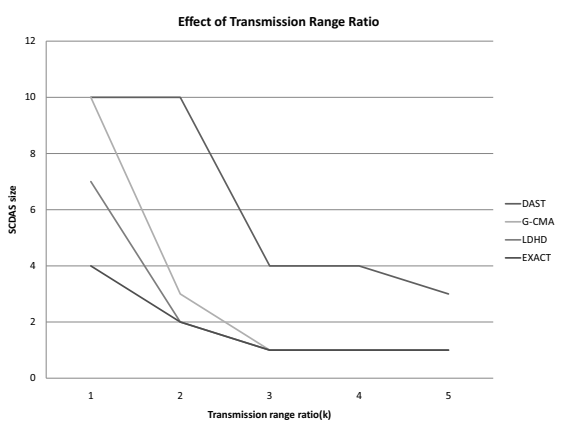

Fig. 3. Different Transmission Ratios, $N=50$

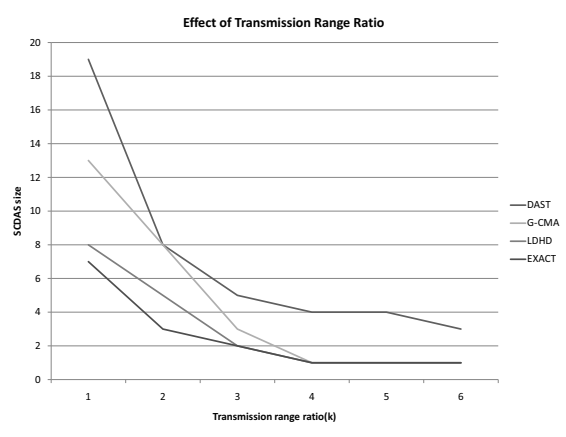

Fig. 4. Different Transmission Ratios, N=100

Fig 1 shows the difference in the size of the strongly connected dominating sets constructed by each of the four approaches. Obviously, DAST constructs the largest such set for all values of $N$, whereas LDHD is the closest to the optimal solution. Similar results are shown in each of the above figures. For all area widths and for all values of $k$, LDHD constructs the smallest set.

In all the conducted experiments the size of our computed solution was never larger than 1.75 optimum, while the sets constructed by the two other heuristics were in the range: 2.5 to 5 times optimum.

\section{CONCluding Remarks}

The problem of computing a virtual backbone in a wireless ad-hoc network is modeled by the NP-hard Strongly Connected Dominating-Absorbent Set problem. Several time-efficient approximation algorithms have been proposed. This paper sudied a simple and highly efficient approach that can deliver solutions whose size is much closer to optimum than solutions of the known published algorithms.

More work is still needed on the virtual backbone problem to incorporate real constraints, due mainly to the dynamic nature of ad-hoc networks. Energy and mobility are just examples of factors to take into consideration. Moreover, the connected dominating set model may also need further refinements. A dominating set of small diameter might be better (for routing purposes) than a minimum dominating set of a larger diameter.

As we consider all the various constraints, we should not neglect the need for highly efficient simple algorithms. Simplicity of an algorithm may play an important role, especially on nodes of mobile/sensor networks, which tend to have limited computational resources.

\section{ACKNOWLEDGMENT}

The authors would like to thank Mr. Ali Ghrayeb for his great help with the experimental study.

\section{REFERENCES}

[1] J. Wu: Extended Dominating-Set-Based Routing in Ad Hoc Wireless Networks with Unidirectional Links. IEEE Transactions on Parallel and Distributed Computing. 22, 327-340 (2002)

[2] F. Dai and J. Wu: An Extended Localized Algorithms for Connected Dominating Set Formation in Ad Hoc Wireless Networks IEEE Transactions on Parallel and Distributed Systems. 15, 902920 (2004)

[3] M.T. Thai, R. Tiwari, and D.Z Du: On Construction of Virtual Backbone in Wireless Sensor Networks with Unidirectional Links IEEE Transactions on Mobile Computing. 7, 1098-1109 (2008)

[4] M. Park, C. Wang, J. Willson, M. T. Thai, W. Wu, and A. Farago: A Dominating and Absorbent Set in Wireless Ad-hoc Networks with Different Transmission Range In:8th ACM International Symposium on Mobile Ad Hoc Networking and Computing, Canada (2007) 
[5] M. T. Thai, F. Wang, D. Liu, S. Zhu, and D. Z. Du: Connected Dominating Sets in Wireless Networks with Different Transmission Ranges IEEE Transactions on Mobile Computing. 6, 721-730 (2007)

[6] D.-Z. Du, M. T. Thai, Y. Li, D. Liu, S. Zhu,: Strongly Connected Dominating Sets in Wireless Sensor Networks with Unidirectional Links Lecture Notes in Computer Science. 3841, 13-24 (2006)

[7] Deying Li, Hongwei Duc, Peng-Jun Wan: Construction of strongly connected dominating sets in asymmetric multihop wireless networks Theoretical Computer Science. 410, 661-669 (2009)

[8] V. Bharghavan, B. Das: Routing in ad hoc networks using minimum connected dominating sets IEEE International Conference on Communication. 1, 376-380 (1997)

[9] Yuanzhu Peter Chen, Arthur L. Liestman: Approximating minimum size weakly-connected dominating sets for clustering mobile ad hoc networks In:3rd ACM International Symposium on Mobile Ad Hoc Networking and Computing, Switzerland (2002)

[10] B. Ramamurthy, J. Iness, B. Mukherjee: Minimizing the number of optical amplifiers needed to support a multi-wavelength optical LAN/MAN IEEE International Conference on Computer Cоттиnications. 1, 261-268 (1997)

[11] A. Salhieh, J. Weinmann, M. Kochha, L. Schwiebert Power efficient topologies for wireless sensor networks International Conference on Parallel Processing. 1, 156-163 (2001)

[12] R. Sivakumar, B. Das, V. Bharghavan: An improved spine-based infrastructure for routing in ad hoc networks In:IEEE Symposium on Computer and Communications, Greece (1998) 